\title{
Limit Load Analysis for Branch Pipe Tee Connection under Internal Pressure and In-Plane Bending Moment
}

Abstract

\author{
Mani $\mathbf{N}^{1}$, Thanigaiyarasu $\mathbf{G}^{2}$ \\ ${ }^{1} \mathrm{PhD}$ Scholar, ${ }^{2}$ Professor \\ CEG Campus, Anna University, Chennai, India \\ e-mail: nmani_75@yahoo.com
}

The present work presents plastic limit load solutions for branch pipe tee connection under internal pressure and in plane bending moment based on detailed three dimensional finite element limit analysis using elastic - perfectly plastic materials. To assure reliability of the FE limit loads, modeling issues are addressed first, such as the effect of kinematic boundary conditions and branch pipe geometries on the FE limit loads. Several models of branch pipe tee connection are meshed with shell elements and submitted to internal pressure with end in plane bending moment. Results are compared with lower and upper bound analytical solutions and experimental results reported in the literature. Computations with 20 noded elements are proposed to validate this analysis.

Key words: Finite ElementAnalysis

\section{NOMENCLATURE}

Mo Limit moment of straight pipe

ML In-plane moment of branch pipe

Po Limit pressure of straight pipe

PL Limit pressure of branch pipe

$R$ Mean radius of main (run) pipe

$R$ Mean radius of branch pipe

$T$ Thickness of main pipe

T Thickness of branch pipe

$\sigma_{o}$ Limiting strength of an elastic-perfectly plastic material

\section{INTRODUCTION}

Standard design rules are based on three principal studies: elastic studies, limit analysis and elastic with hardening plasticity analysis. The first is the most developed because of its relative simplicity and its universal use. Elastic with hardening plasticity analysis is nowadays feasible thanks to the numerous non linear finite element codes and powerful computers. Limit analysis has often been forgotten perhaps because, in the past, it could only be used for special studies where upper bound and lower bound theorems could solve analytical problems.

Information on the limit load of piping components is important in structural integrity assessment. Such information is a direct input to estimate the maximum load carrying capacity of piping components [1]. Furthermore based on the reference stress approach [2], it can be used to estimate creep rupture and non-linear fracture mechanics parameters. Due to its significance, information on limit loads for typical piping components with or without defects is widely available. As branch junctions are widely used in plants such as nuclear reactors (steam generator), numerous works on plastic limit analysis of branch junction have been reported. Although some analytical works are available [3-5], works based on non-linear finite element analysis are increasingly popular, due to complexities associated with the geometry and loading conditions.

Two issues need to be resolved on plastic limit analyses of branch junctions. As works based on nonlinear FE analysis are popular, FE modeling issues should be resolved, but not much information has been given in the literature. As the second issue, for practical application, reliable limit load solutions need to be developed in a closed form. Although, some solutions are available for branch junctions, the reliability of existing solutions needs to be checked.

The present work presents plastic limit load solutions for branch junction under internal pressure and in-plane bending moment based on detailed three-dimensional (3D) FE limit analyses using elastic-perfectly plastic materials. The branch junction considered in the present work is thin walled cylinders. ANSYS 10.0 Finite Element code is used to find the limit load of complex structures: branch pipe tee connections submitted to internal pressure and in-plane bending moment using three nodded shell elements (shell 163). Results are compared with existing solutions and experimental results that can be found in literature.

\section{EXISTING LIMIT LOAD SOLUTIONS}

Consider the branch tee junction depicted in fig 1. For the main (run) pipe, the mean radius and thickness are denoted by $R$ and $T$ respectively and for branch pipe $r$ and $t$ respectively. It is assumed that the branch junction has no weld or reinforcement around the intersection. In the literature there are several limit load solutions for branch connections [6]. Among these selected solutions, which are belived to be most reliable, are reviewed here. 
For internal pressure, Budden and Goodall [7] proposed the following regression equation for the limit pressure, $\mathrm{PL}$, based on FE date

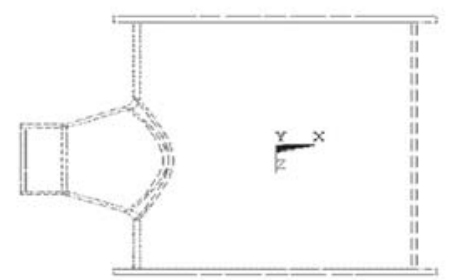

Fig. 1 Branch Tee Pipe

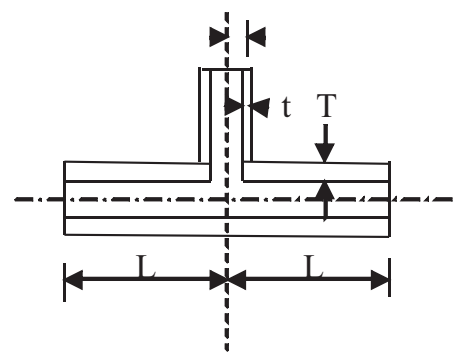

Fig.2. Schematic of Branch Tee Pipe

PLR $\quad A[1+B(1-r / R][1-D(0.5-T / R) 2]$

$----=$

боT $\quad[1+C(1-t / T)]$

with $A=0.641 ; B=0.908 ; C=0.608 ; D=1.422$.

Although budden and Goodal checked the validity of this solution by comparison with analytical solutions by Robinson [5] and FE data, the main concern seemed to be applications to thick wall tubes. The variation of results are shown in fig. 3 for $r / R=0.6$. It can be seen that, in the limiting case of $r$ ? $0(t ? 0)$, the above solution does not always recover the plastic limit pressure for the tube based on the Von-Mises condition, Po:

PoR 2

боT $\quad$ 3

Xuan et al.[4] proposed the following analytical limit pressure solution, $\mathrm{PL}$ :

PLR

(3)

бо $\quad(0.25-0.5 \mathrm{~h} 1+\mathrm{h} 12+0.79 \mathrm{~h} 22) 0.5$

with $h 1=1+(0.145 \mathrm{kAvBf} 2+0.3185 \mathrm{~A} 2 \mathrm{f} 1)(1-\mathrm{C} / \mathrm{AB}) 2$

$\mathrm{h} 2=0.175 \mathrm{kAv} B((1-\mathrm{C} / \mathrm{AB}) 2 \mathrm{f} 2$

f1' $1+$ XA4; f2' $\pi / 2(1-0.1875 \mathrm{~A} 2) ; \quad k=\quad \begin{aligned} & 1 \\ & ---\end{aligned}$
$1+\mathrm{C} 3$

$A=r / R ; \quad B=2 R / T ; \quad C=t / T$

This equation derived from force equilibrium between the limit load and the internal force acting on the intersecting line between the run and branch pipe. Xuan et al. compared with experimental data and with FE data and found overall good agreement. From the above two solutions equation (1) always gives a lower limit load than eqn.(3). The variation of limit load results are shown in fig. 4 for $r / R=0.6$. In particular as eqn.(1) does not give the correct limit load for the limiting case of $r$ ? 0 , the difference between eqn.(1) and eqn.(3) increases as $r / R$ decreases. The limit loads from the above two are similar, bur those from eqn. (3) are slightly higher.

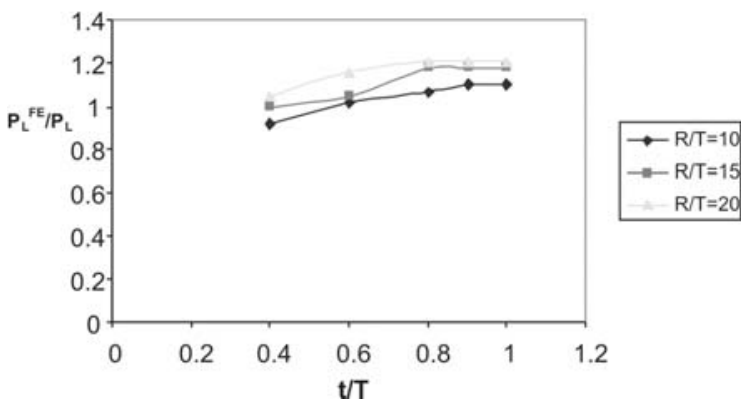

Fig.3. The Variation of Limit Load Solution [7] Under Internal Pressure

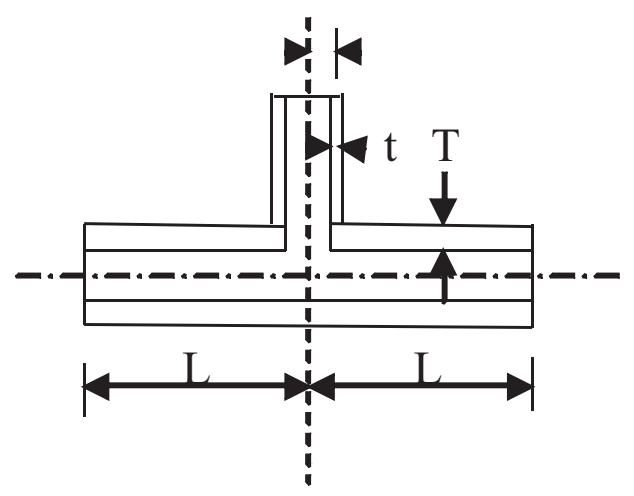

Fig.4. The Variation of Limit Load Solution [4] Under Internal Pressure

For in-plane bending moment (with the bending moment applied to the end of branch pipe), the solution by Xuan et al.[3] is given by

$\mathrm{ML}$

$----=$

$\pi / 2$

(4)

Mo $\quad \mathrm{C}[(\mathrm{f} 1 \mathrm{~A}+0.455 \mathrm{f} 2 \mathrm{kv} B) 2+0.2385 \mathrm{~B} f 22 \mathrm{k} 2] 0.5$

and $f 1, f 2, k, A, B$ and $C$ are given in eqn.

$\mathrm{Mo}=4 \sigma 0 \mathrm{r} 2 \mathrm{t}$ 
(5)

The above solution was derived from force equilibrium between the limit load and the internal force acting on the intersecting line between the main and branch pipes. In the limiting case of $\mathrm{T}$ ? 0 or R? 0 , eqn.(4) recovers the exact limit moment for the branch pipe. The general trend is that for smaller branches $(r / R=0.3)$, the limit load for the branch junction is the same as that for the branch pipe, and weakening effects occur only for for larger branches ( $r / R 0.7)$. The limit load for the branch pipe tends to saturate. The variation of limit load results are shown in fig. 5 for $r / R=0.9$. The results are compared with experimental data with FE data and found overall good agreement.

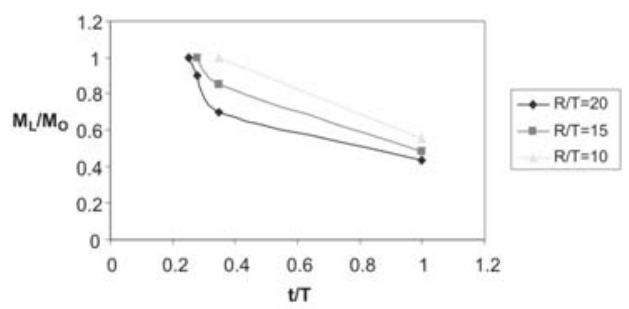

Fig.5. Variation of Limit Load Solution [3] Under In-Plane Bending Moment

Y.J.Kim et.al [8] proposed the following equations for branch junction under internal pressure and in-plane bending moment:

$$
\begin{aligned}
& \text { PLR } \\
& \text { (6) } \\
& \text { боT } \quad(0.25-0.5 h 1+h 12+0.79 h 22) 0.5 \\
& \text { with } Q=1.32(A=0.636) 2+0.906 \text { and } k=\frac{1}{0.78(C+0.55) 3}
\end{aligned}
$$

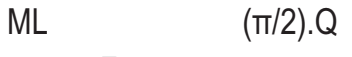$$
\text { ---.-- = }
$$$$
\text { (7) }
$$$$
\text { 40o r2t } \quad \mathrm{C}[(\mathrm{f} 1 \mathrm{~A}+0.455 f 2 \mathrm{kv} B) 2+0.2385 \mathrm{Bf22k} 2] 0.5
$$$$
\text { where the factor } Q \text { is given by }
$$

$\mathrm{Q}=-1.11(\mathrm{C}-0.7) 2+1.18$.0ther factors $\mathrm{h} 1, \mathrm{~h} 2, \mathrm{f} 1, \mathrm{f} 2, \mathrm{~A}, \mathrm{~B}$ and $\mathrm{C}$ are the same as those given in eqn.(1). Noting that the value of Qin the limiting case oft $/ T$ ? 0 or r/R? 0 approaches 0.64 .

\section{FINITE ELEMENT LIMIT ANALYSIS}

3-D elastic perfectly plastic $\mathrm{FE}$ analysis of the branch pipe tee junction depicted in fig.1 were performed using ANSYS. It is assumed that the branch junction has no weld or reinforcement around the intersection. Regarding the axial length, the half length of the run pipe is denoted as $L$ and the length of the branch pipe as I. The geometric variables $R, T, r, t, L$ and I were systematically varied with in the range $\quad 0.4=(r / R, t / T)=1.0$ and $10=R / T=20$. Note that such ranges correspond to thin-wall cylinders. Materials are assumed to be elastic perfectly plastic. Symmetry conditions were fully utilized in FE models to reduce the computing time. SHELL163 is a 4-node element with both bending and membrane capabilities. Both in-plane and normal loads are permitted. The element has 12 degrees of freedom at each node: translations, accelerations, and velocities in the nodal $x, y$, and $z$ directions and rotations about the nodal $x, y$, and $z$-axes. Fig. 6 shows typical FE meshes, employed in the present work

Regarding loading conditions, both internal pressure and in-plane bending moment were considered. For internal pressure, pressure was applied as a distributed load to the inner surface of the model and at the end of branch pipe moment is applied. Due to symmetry, only quarter model was used for internal pressure and halfsymmetry model was used application of both internal pressure and in-plane bending moment. For in-plane bending cases, the nodes at the end of the branch pipe were constrained through multi point constraint (MPC) option with in ANSYS.

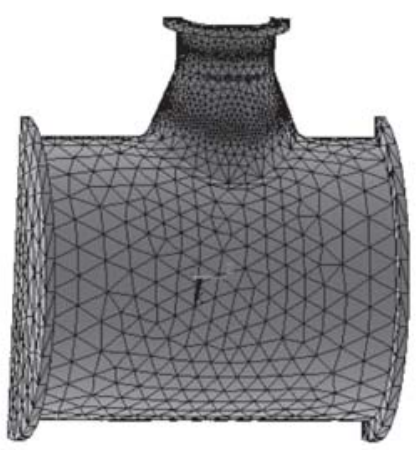

Fig.6.Finite Element Mesh

\section{RESULTS AND DISCUSSION}

\section{A. Internal Pressure}

For the data indicated as Budden et.al, the FE limit loads are normalized with respect to eqn.(1), whereas for those indicated as Xuan et.al. with respect to eqn.(3).For the data indicated as budden, the values are overall higher than unity by upto $40 \%$, suggesting that eqn.(1) gives overall lower limit loads than $\mathrm{FE}$ results. On the other hand Xuan et al. the values can be lower than unity upto $20 \%$, suggesting eqn((3) can give higher limit loads than FE results. It can be also noted that the effect of $R / T$ is less significant for Xuan et al. data than Budden et al. data. 
Whereas Y.J.Kim et al. datas respect to eqn.(6) are 10\% less than FE data for all the cases considered and good agreement with the experimental data. Fig 7(b) compares the Y.J.Kim et al. limit moment solution for various cases. The FE limit loads in fig 7 (b) are normalized with respect to the eqn.(6) and thus the proximity of the data to unity indicates the accuracy.

\section{B. In-plane bending moment}

FE limit loads are normalized with respect to the analytical expression, eqn.(4).FE results are always higher than eqn.(4) by upto $30 \%$ and thus gives conservative moments. It should be noted that that Xuan et al. compared the limit loads from eqn.(4) with experimental results and found that predictions according to this equation were slightly lower than the experimental data by upto $5 \%$.One intersecting point is that, for the comparison, they used the flow strength as 1.25 times the yield strength but experimental loads were measured using twice elastic slope method from load-displacement curves. If they had used the yield strength as the flow strength, the predictions would be lower than the experimental results by upto $30 \%$, which is consistent with the present findings. Whereas Y.J.Kim et al. solution gives lower limit moments than FE data by less than $10 \%$ for all the cases considered. Fig.7(a) compares the limit moment solution with the FE results for various cases.

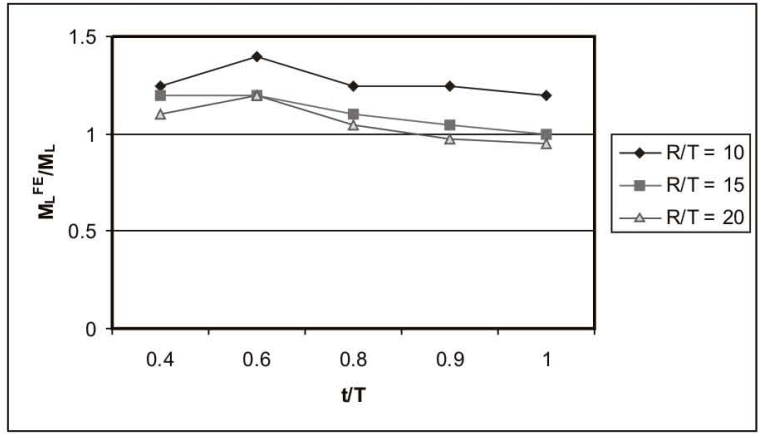

Fig. 7 (a) Variations of Limits of FE Limit Loads Under In-Plane Bending

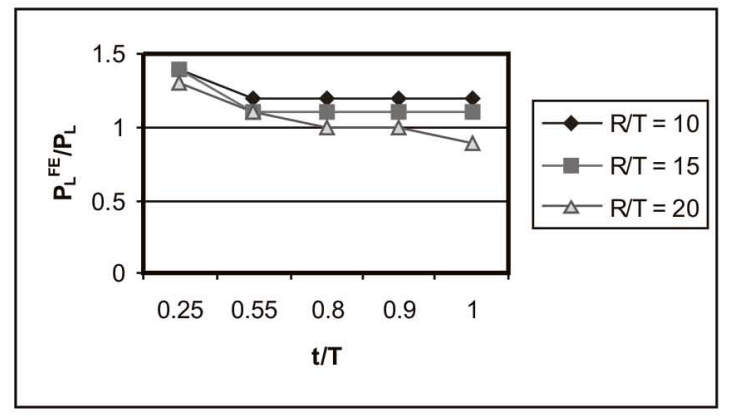

Fig.7 (b) Variations of Limits of FE Limit Loads Under Internal Pressure

\section{CONCLUSION}

The present work presents plastic limit loads solution for branch pipe tee connections under internal pressure and in-plane bending moment, based on detailed FE limit analysis using elastic-perfectly plastic materials. To assure reliability of FE limit loads, modeling issues are addressed first, such as the effect of boundary conditions and branch junction geometries on the FE limit loads. Based on systematic $\mathrm{FE}$ analysis for branch junctions with $0.4=(r / R, U / T)=1.0$ and $10=R / T=20$ under internal pressure and in-plane bending moment, the FE limit loads are determined and compared with existing limit load solutions .For internal pressure, the solution by Budden et al.overall underestimates by upto $40 \%$.For in-plane bending, it is found that the analytical solution given by Xuan et al. underestimates the FE limit loads by upto $30 \%$. Based on experimental results, FE limit load solutions are agree well and Y.J.Kim et al. solution underestimates by less than $10 \%$ for all cases considered.

\section{REFERENCES}

[1]. Kanninen MF et al. Instability predictions for circumferntially cracked type-304 stainless pipes under dynamic loading, EPRI NP-2347.

[2]. Ainsworth RA. The assessment of defects in structures of strain hardening materials. Eng.Fract.Mech 1984;19:633-42

[3]. Xuan et al. Limit laod anaysis for the piping branch junctions under in-plane moment. Int. J Mech Sci 2006;48:460-7.

[4]. Xuan et al. Limit laod anaysis for the piping branch junctions under internal pressure.Nucl Eng Design 2003;224:1-9.

[5]. Robinson M. Lower bound limit pressures for the cylinder-cylinder intersection: a parametric survey. J Pressure Vessel Tech 1978;100:119-20.

[6]. Miller AG. Review of limit loads ofstructures containing defects. Int J Pressure Vessel \& Piping 1988;32:191-207.

[7]. Budden et al.Creep life of pressure vessel branch connections : the reference stress approach.Nuclear Electric report TD/SID/REP/0123, 1990

[8]. Y.J.Kim et al. Limit loads for thin walled piping branch junctions under internal pressure and in-plane bending, Int J Pressure Vessel \& Piping, 2006. 